\title{
Analysis of The Influence of Nursing Intervention on the Mental Health of Maintenance Hemodialysis Patients
}

\author{
Xingli $\mathrm{He}^{*}$ \\ Hemodialysis Room, Nanzhou People’s Hospital of Qianxi, Qianxi, Guizhou 562400, China
}

\begin{abstract}
Objective: To explore the effect of nursing intervention on the mental health status of patients through maintenance of hemodialysis patients with psychological counseling, psychological health education, treatment and other aspects while promoting the treatment of the disease. To study the effect of nursing intervention on the self-behavior management and anxiety in the patients. Methods: The psychological status of the patients was assessed through questionnaires which conducted by nurses with patients. The nurses asked for cooperation of patients and led them to complete a classified questionnaire. According to different psychological problems, the corresponding nursing measures were taken. Results: Most of the patients with maintenance hemodialysis had different levels of depression, fear, paranoia, interpersonal sensitivity and other psychological problems. Conclusion: Patients, who had psychological problems, received nursing intervention through nurse-patient relationship in the maintenance of hemodialysis patients. Self-care education could improve patients' self-management, which could reduce the patients' anxiety and depression. Their mental health had significantly improved. The treatment of disease ensured long-term survival, prolonged their life cycle and improved quality of life of patients.
\end{abstract}

\section{KEYWORDS}

Maintenance hemodialysis

Nursing intervention

Mental health

\section{Introduction}

Clinically, hemodialysis patients would suffer from life style and psychological pressure. Once patients started dialysis treatment, they must rely on dialysis machine to sustain their lives. Thus, the maintenance hemodialysis patients need a long-term continuous hemodialysis treatment. However, different degrees of adverse reactions and complications would occur during hemodialysis, and severe mental disorders would easily be developed. Psychological activities of patients could affect their physiological function, and it may lead to reduced body's immune system which is a disease prognosis [1]. The increasing number of hemodialysis patients has demanded more atten-

\section{Copyright $\odot 2016$ Xingli He}

doi: $10.18686 /$ jn.v5i2.13

Received: June 23, 2016; Accepted: August 4, 2016; Published online: August 26, 2016

This is an open-access article distributed under the terms of the Creative Commons Attribution Unported License (http://creativecommons.org/ licenses/by-nc/4.0/), which permits unrestricted use, distribution, and reproduction in any medium, provided the original work is properly cited.

${ }^{\star}$ Corresponding author: Hemodialysis Room, Nanzhou People’s Hospital of Qianxi, Qianxi, Guizhou 562400, China. E-mail: hexingli123y@ sina.com tion on the psychological problems of patients. To improve the quality of dialysis in hemodialysis patients, the medical staffs and patients should participate in care and treatment of the disease. From June 2011 to June 2014, there were 1121 cases which received hemodialysis treatment in our hospital. The study focused on 100 patients from 1121 cases. In order to have a comprehensive understanding of their physiological responses and psychological barriers, questionnaire survey was necessary and nursing intervention should be carried out. Nurse-patient interaction should be carried out in the maintenance of hemodialysis patients, which could effectively affect patients' self-management and the change of self-behavior. Subsequently, their rehabilitation could rapidly be promoted. Nurse-patient interaction was carried out in maintenance of hemodialysis patients in our study and it has achieved favorable results.

\section{Materials and methods 2.1. Clinical data}

The psychological barriers of 100 cases of hemodialysis patients mainly included interpersonal sensitivity, paranoia, fear, anxiety and depression. The patients were in a stable state, had a clear sense of consciousness and no 
serious complications. There were 65 male and 35 female among these 100 patients whose age ranged between 26 to 74 years old. The patients received dialysis 2 to 3 times a week, 4 hours each time, and for 7 to 52 months.

\subsection{Survey method}

The questionnaire also called an inquiry form. It is a printed matter in the form of the problem. The principle of the questionnaire is every question and answer in the questionnaire should contribute to the required information or serve a specific purpose. In order to obtain the required information, several questions are posed. The questionnaire which was used to assess the psychological status of patients was supplied to the patients with cohesive guidance language. Then, patients will be asked and guided to fill in the questionnaire. The content of questionnaire mainly involved in the problem of patients with mental disorders, which is interpersonal sensitivity, anxiety, fear, paranoia, anxiety, despair, and others. The questionnaires were handled by professional nurses.

\subsection{Nursing intervention measures}

It is necessary to establish a good relationship between nurses and patients. When nurses attend to patients, they should stand in the perspective of patients, put themselves for the sake of patients, pay attention to the patients and respond in a gentle manner with patients. First of all, the nurses in the department of hemodialysis should provide patients with intervention and guidance in between and after hemodialysis. Then, the nurses should follow-up with observation before the final evaluation is given. Patients should strictly follow the requirements of the medical staffs and dialysis on time to improve the quality of life and survival.

Successful stories and experience of other patients should be shared to the patients. This will enable the patients to have confidence on the treatment, actively cooperate with medical staffs thus improving compliance. At the same time, patients should attend mental health education and obtain support from family members. Nursing staffs should treat patients with enthusiasm and respect. They should also explain the problems of the disease to the patients, and maintain a cheerful environment for a stable and speedy recovery. Nursing staffs should improve their own service quality to correct the non-cooperative behavior of patients which was due to lack of knowledge over the disease.

A good relationship between patients, doctors and nurses should be established. The patients should be given psychological guidance with a purpose. Family members of patients should always comfort and encourage patients, which could enhance survival consciousness and extend the survival cycle. Finally, nurse staffs should encourage and guide patients to participate in social and various sports activities, which could help the patients to over- come the psychological barriers. Communication with the patients with same disease could help patients to ease their inner anxiety. The staffs should help patients to form other interest, which can improve their social ability, reduce the psychological pressure of patients, and promote their physical and mental health $[2,3]$.

\section{Results}

Nursing intervention on maintenance hemodialysis patients had achieved a favorable result. The situation of before and after nursing intervention are shown in the following Table 1:

Table 1. Nursing intervention on maintenance hemodialysis patients.

\begin{tabular}{ccccc}
\hline Mental disorders & $\begin{array}{c}\text { Before nursing } \\
\text { intervention }\end{array}$ & $\begin{array}{c}\text { After nursing } \\
\text { intervention }\end{array}$ & $X^{2}$ & $p$ \\
\hline $\begin{array}{c}\text { Interpersonal } \\
\text { sensitivity }\end{array}$ & 23 & 0 & 11.93 & $<0.01$ \\
Anxiety & 45 & 9 & 21.86 & $<0.01$ \\
Fear & 78 & 15 & 37.73 & $<0.01$ \\
Paranoid & 75 & 21 & 19.63 & $<0.01$ \\
Depression & 87 & 19 & 37.45 & $<0.01$ \\
Angry & 65 & 17 & 43.38 & $<0.01$ \\
Desperate & 87 & 24 & 51.05 & $<0.01$ \\
Worry & 90 & 28 & 53.35 & $<0.01$ \\
\hline
\end{tabular}

Note: There was statistical significant difference between before and after the psychological intervention $(p<0.05)$.

\section{Discussion}

Clinically, maintenance of hemodialysis patients who are in acute conditions or unable to perform daily life activities require a long period of treatment and rehabilitation. Therefore, patients should be ready to face the pressure of life, economy and other aspects despite of going through anxiety, tension, depression and other negative emotions. The nurse-patient interaction and self-care education included cultivating of self-care ability of patients and assisting patients to go through the crisis.

The principle should be implemented with its intensity varying from person to person. Then, the patients shall be guided gradually to learn self-care. Based on the actual situation of patients, patients are guided to master the nursing skills of relevant diseases which may accelerate the rehabilitation process and reduce the complications.

\subsection{Analysis of psychological states in patients with hemodialysis \\ 4.1.1. Inferiority feelings}

This was due to dry skin, pigmentation, peeling, body edema, hair loss and other physical changes which are the side effects of the prolonged dialysis received by maintenance hemodialysis patients as part of the body's physiological function is declined. The deterioration of own family and 
social values resulted in inferiority complex [4].

\subsubsection{Anxiety and fear}

Anxiety and fear are the common psychological reactions in maintenance hemodialysis patients. The patients developed fear and anxiety especially in the early stage of hemodialysis. They are not aware of the need of hemodialysis machine, the establishment of vascular access, the strange environment of hemodialysis room and the development of their own disease. In addition, they also worried about the safety of the nurse's skill on venipuncture and feared of unnecessary pains [5].

\subsubsection{Pessimism and despair}

Besides kidney transplantation, the only way to maintain lives of patients is hemodialysis. However, hemodialysis is just an alternative therapy and it could not stop the development of the disease. Therefore, patients would develop a psychology of pessimism and despair. Blood dialysis is a painful process, with regular vein piercing, and patients need to repose four hours with each hemodialysis.

Patient's psychological would give rise to a certain pressure, and a variety of hemodialysis complications, such as headache, nausea, fever and other complications that patients suffered from a lot of pains, which made them loss of confidence. During dialysis process, the intake of water, salt, food would be restricted. In some worse cases, despaired patients with pain and treatment even develop the idea of committing suicide [6].

\subsection{Psychological nursing of maintenance hemodi- alysis patients \\ 4.2.1. Establishing a good relationship between doctor and patient}

Nursing staffs should evaluate the adverse psychological reactions based on the patient's personality before reducing the patient's fear, tension, anxiety and other adverse psychological. Patients sleep and diet should also be taken into care. Thus, the cognition of hemodialysis would be enhanced, while the trust towards doctors and nurses would get better.

\subsubsection{Enriched communication between nurses and patients}

Nurses should be polite to receive patients who enter dialysis room, and patiently explain hemodialysis to patients with their own language and technical skills. Nursing staffs should patiently listen to patients, treat patient with careful and thoughtful, and also encourage patients to overcome the disease. This would help patients to increase their con- fidence.

\subsubsection{To improve the recognition of patients with he- modialysis}

The patients have different cognition of the disease due to the difference in social experience and cultural level. Nursing staffs should introduce the importance of hemodialysis, its application, the development of the disease, the treatment of complications to patients and also guide them to have a reasonable exercise and diet, which indirectly promotes the recovery of patients.

\section{Conclusions}

Through the comprehensive system of care, the patients with mental problems could be treated and the medical disputes could be reduced. Nursing staffs should also enrich their knowledge, improve skills of venipuncture, and solve the psychological problems of patients with a sincere attitude and warm service. Apart from regular concern over the disease, the nurses should also pay attention to patients' mental health problems and their psychological needs to improve their personality [7].

In summary, nurse-patient interaction should be carried out in maintenance hemodialysis patients. Self-care education could improve patients' self-management and selfefficacy, which can reduce the patients' anxiety and depression, and patients will be able to consciously manage their diseases and promote their rehabilitation.

\section{References}

1. Liu J. Effect of intensive nursing intervention on the incidence of fistula complications in maintenance hemodialysis patients. Contemp Nurs. 2013;(1):125-127.

2. Wang $X$. Analysis of the influence of nursing intervention on the anxiety state of maintenance hemodialysis patients. J Chifeng Instit (Nat Sci Ed). 2014;(24):66-68.

3. Du Y, Yang J, Tu Y. Effect of nursing intervention on the depressive state of maintenance hemodialysis patients. Mod Med. 2011;(1):101-104.

4. Sun GY, Xu PL, Zhu CY. Effect of nursing intervention on dry weight of maintenance hemodialysis patients. Nat Med Front China. 2012;(10):71-73.

5. Wu X, Huang J. Effect of early nursing intervention on the incidence of arteriovenous fistula in maintenance hemodialysis patients. Mod Dr China. 2014; (1): 96-99.

6. Chen YM. Effect of psychological nursing intervention on maintenance hemodialysis patients. J Nurs Train. 2012;(18):1699-1700.

7. Chang $M$, Dai $H$, Ji X, et al. The influence of nursing intervention on maintenance hemodialysis patients. Nurs Pract Res. 2012; (20):38-39. 